Research Article

\title{
Portable Low-Frequency Noise Reduction Device for Both Small Open and Closed Spaces
}

\author{
Chao Wang, ${ }^{1}$ Haiyang Gao,, ${ }^{1,2}$ Liyao Yu, ${ }^{1}$ Tiantian Yu, ${ }^{1}$ Wenhui Yan, ${ }^{1}$ and Qiumeng Xue \\ ${ }^{1}$ Key Laboratory for Aerosol-Cloud-Precipitation of China Meteorological Administration, \\ Nanjing University of Information Science and Technology, Nanjing 210044, China \\ ${ }^{2}$ School of Atmospheric Physics, Nanjing University of Information Science and Technology, Nanjing 210044, China
}

Correspondence should be addressed to Haiyang Gao; yuanzineng@126.com

Received 17 April 2015; Revised 6 September 2015; Accepted 8 September 2015

Academic Editor: Carlo Trigona

Copyright (C) 2016 Chao Wang et al. This is an open access article distributed under the Creative Commons Attribution License, which permits unrestricted use, distribution, and reproduction in any medium, provided the original work is properly cited.

\begin{abstract}
Noise pollution has been given more attention due to its negative impacts on human health and disease. The portable low-frequency noise reduction device we developed in this research can provide an effective way for solving low-frequency noise pollution problem in the small space. This work describes the design principle and the prototype structures for two versions of V1.5 and V2.0 and builds the noise test systems for small spaces, respectively. These devices, installed on the outer surface of the small spaces, can automatically identify the noise spectrum and implement noise reduction by means of the active noise control (ANC) technology. The testing results indicate that the noise can be reduced $12 \mathrm{~dB}$ in the range of $250 \mathrm{~Hz} \sim 400 \mathrm{~Hz}$ for the small closed space while, for the small open space, the best effect of $5.88 \mathrm{~dB}$ occurs in the optimal frequency of $450 \mathrm{~Hz}$. These effects will be weakened with the increasing distance away from the source and show the obvious axisymmetric distribution in the inverted cone space.
\end{abstract}

\section{Introduction}

Although the noise pollution has become one of the major environmental issues, it obtains a low priority compared with other pollutions including the air, water, and solid waste pollutions. Regarding this issue, the world health organization and the European Union cooperative research center released a report studying on the impact of noise on health "Disease Burden Caused by Noise Pollution." According to research results, the excessive exposure in noise pollution can produce the serious influence sleep and mental health but also cause learning disabilities and diseases such as tinnitus, and it can increase the risk of heart disease. Therefore, how to reduce noise pollution has become a main problem of environmental pollution control in this century.

The noise reduction technology can be divided into two categories including the passive and the active noise control (ANC) technology [1-4]. For the mechanism of passive noise control technology, the acoustic materials or structures, in general, are used to achieve the sound absorption, sound insulation, muffler, vibration isolation, and damping vibration. This technology is capable of reducing the middle and high-frequency noise. While the ANC technology provides a good solution on the low-frequency noise control. Based on the interference principle, the original wave from the noise source can be weakened by a secondary wave with opposite phase and the same propagating direction. As a result of the development of computer technology and the signal processing technology, the ANC technology has entered a rapid development period. The emergence of the high constant power speaker, secondary sound source, transducer, and related signal processing devices plays an important role in making the ANC technology enter the practice stage [5]. Compared with the passive noise and vibration control, the ANC has a lot of advantages such as the ability to work in the low frequency, pertinence and small in size, flexible, and convenient.

The concept of noise reduction based on acoustic interference effect is first put forward by Rayleigh, while German physicist Lueg fabricates a real device which is published as one US patent and one paper [6]. Olson and May, two engineers in RCA company laboratory, provide an electronic speaker seen as an active noise control system [7]. By using a feedback control system, Olson and May put this device 
in the seat above the back of a chair to reduce the noise of the person nearby. Conover proposed the active noise control used for transformer noise control [8]. It is only suitable for the space of less than 20 degrees off axis range within a certain amount. After 1980s the digital signal processing technology and device has a significant development as well as the adaptive active noise control technology. Salikuddin and Zinn design a set of secondary sound source array between the propeller and the engine room, and this cabin noise reduction achieved a great effect finally [9]. Anderson et al. propose a multiple input and multiple output of adaptive active noise cancellation system [10]. Zhang et al. propose a nonlinear ANC system by introducing an appropriate secondary source [11].

In recent years, ANC technology has been applied in the manufacture and development of headphones. Liu et al. design an active noise reduction controlling method and system applied to the headphones [12]. Moon et al. analyze the performance of the adaptive feedback active noise control (FBANC) based on earmuffs system [13]. Miyazaki and Kajikawa apply a virtual sensing technique to a head-mounted ANC system, which can achieve higher noise reduction at the desired locations by measuring the system models from physical sensors to virtual sensors [14]. Zhang and Qiu propose a systematic analysis to predict the performance of a typical feedforward single channel ANC headset in terms of the delay, especially the noncausal delay caused by different noise coming directions [15]. Headphones with adaptive feedback ANC show a good performance if the secondary path is well known. The secondary path however changes considerably if the headphones are lifted. Guldenschuh and Callafon develop a cost-efficient algorithm in the time-domain to detect and react to these changes in order to keep the ANC system stable [16].

With the development of the electronic technology, computer algorithm, and the signal processing technology, the application areas of ANC technology are also further expanded. Wang et al. apply active noise control technologies in the exhaust port of a vacuum cleaner to reduce lowfrequency noise [17]. Pan et al. report the progress of a couple of practical ANC installations in vehicle cabins [18]. Kwon and Park propose an active window system, which uses a feedforward control method for active noise control, to reduce the exterior noise sources, such as traffic noise and construction noise which enter rooms through open windows used for natural ventilation [19]. Airaksinen and Toivanen present a new method by using numerical acoustical modeling to obtain a local active noise control that is optimal in stochastic environment [20]. Tanaka deals with local as well as global ANC using a parametric array loudspeaker (PAL) possessing intriguing properties: sharp directivity, low sound pressure decay by distance, and capability of steering directivity [21]. Ardekani and Abdulla propose adaptive active control of acoustic noise in free space [22]. In the further work, Ardekani and Abdulla also propose a methodology for developing a family of acoustical signal processing algorithms that are able to control sound in three dimensions and derive an optimal control system for 3D ANC [23].
At present, the ANC research mainly focused on the multichannel adaptive broadband noise cancellation system [24]. Additionally, the use of noise reduction system is developed based on the artificial neural network to solve the multichannel signal processing and the expansion of dead band.

In order to eliminate the influence of secondary sound feedback and improve the stability, the research on the adaptive algorithm is also an important aspect [25]. Petersen et al. propose an inverse modeling of the structure of the active noise canceller [26]. In this structure the cascade and controller consists of two parts as the predictor and the secondary channel of the inverse model of time delay, respectively. Zhang et al. derive an adaptive active nonlinear noise feedback control approach using a neural network [11]. Tarabini and Roure also propose a novel method by using the neural network for active noise control [27]. Zhou et al. develop an iterative learning control (ILC) algorithm for an ANC system with a nonminimum phase secondary path [28]. Ferrer et al. present the implementation of ANC systems over a network of distributed acoustic nodes [29]. Patel and George propose a spline adaptive filter (SAF) based nonlinear ANC system [30].

Although the noise reduction devices, generally, have been successful applied in many areas, the technology has its own limitations and still cannot be available for the civilian areas such as home, school, hospital, and office. Therefore, we provide a portable low-frequency noise reduction device based on the active noise control technique to effectively eliminate the noise emitting from the small space environment. This research will cover primarily the basic principle in Section 2. Sections 3 and 4 provide the structures of three versions and their experimental testing system and method in brief. Section 5 contains the performance analysis on the noise reduction devices and discussions, and a concluding summary in Section 6 completes this paper.

\section{Basic Principle of the Noise Reduction Device}

Figure 1 shows the basic principle of this noise reduction device based on ANC technology. This device is installed on the outer surface of the small spaces requiring noise reduction processing. The small microphone can firstly receive the noise source entering into the spaces and identify its spectrum. By means of ANC technology, the control elements and electroacoustic devices then produce a secondary sound wave. Since the secondary wave has the equal amplitude, opposite phase, and same propagating direction compared with the source ideally, the noise can be weakened to a lager extent along its propagating path based on the interference principle.

The workflow of the noise reduction device is shown in Figure 2. The noise reduction function can be realized by using the integrated circuit which is made of a noise collector, a signal amplifier, an antialiasing filter, an A/D converter, an adaptive active noise controller, a D/A converter, a smoothing filter, an audio amplifier, and a reversed phase noise output module. 


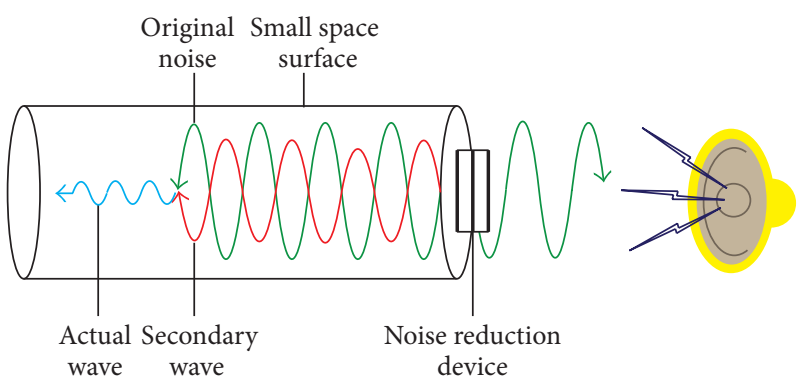

FIgURE 1: The basic principle of the noise reduction device.

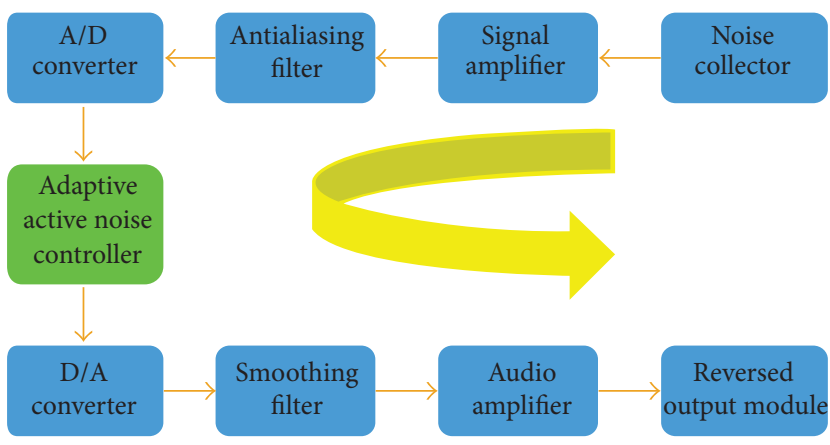

FIGURE 2: The work flowchart of the noise reduction device.

The detailed work processing is described as follows:

(1) Firstly, the noise collector picks up the original noise and sends it to the signal amplifier.

(2) The signal amplifier enlarges the original noise signal and carries it into the antialiasing filter. It is worth mentioning that the amplified signal is capable of making the sound features more obvious for the subsequent processing easily.

(3) The antialiasing filter removes the components with the frequencies more than Fs/2 in the analog signal, in order to ensure the discrete-time signals will not be confused in the spectrum. Then the signal is sent to the $\mathrm{A} / \mathrm{D}$ converter.

(4) The A/D converter converts the analog signal processed by the antialiasing filter into digital signal and takes it to the adaptive active noise controller.

(5) The adaptive active noise controller achieves the function of the active noise reduction processing of the noise signals; more specifically, it can analyze the phase of the digital signal and generate a so-called secondary signal with the equal and opposite phase. This signal is then transmitted to the $\mathrm{D} / \mathrm{A}$ converter.

(6) The D/A converter has the capability of transforming the secondary digital signal into the analog one and delivering it to the smoothing filter.

(7) Due to the fact that the processed analog signal is the staircase signal which may confuse other signals, it needs to be smoothed by the smoothing filter and moved to the audio amplifier again.
(8) The audio amplifier magnifies the smoothing signal and sends it to the opposite phase output unit.

(9) The opposite phase output unit produces another new secondary signal with the same wavelength and direction and the opposite phase. According to the wave interference principle, the original noise can be weakened or eliminated by the new secondary signal.

\section{The Structure of the Noise Reduction Device}

In order to achieve the noise reduction, we design three versions for the noise reduction device including V1.0, V1.5, and V2.0, respectively. The V1.0 device is the first conceptual design in which the application aspects are not considered [31], while both the V1.5 and V2.0 devices are capable of realizing the function of the noise reduction [32]. The detailed design features and functions of V1.5 and V2.0 are given in Sections 3.1 and 3.2.

3.1. The Design of the Noise Reduction Device V1.5. The V1.5 device is designed for realizing noise reduction in the small closed space. The noise comes from out of the space, while the noise reduction process only occurs inside the small closed space. The structure and real picture of the V1.5 device are shown in Figure 3. The V1.5 device is composed of a circular chassis and four-layer clamping groove plates which are joined together by four cylinders, respectively. The eight clamping grooves on the circular base plate allow four same electret pickups set on the corresponding place as the noise collectors and allow four same moving coil loudspeakers set in the other grooves as the opposite phase output units. Two noise reduction circuit boards are fixed on the first and second groove plates, respectively. Both the circuit boards integrate a few electronic elements consisting of the noise collector, the signal amplifier, the antialiasing filter, the $\mathrm{A} / \mathrm{D}$ converter, the adaptive active noise controller, the D/A converter, the smoothing filter, the audio amplifier, and the opposite phase noise output unit. The pickups and loudspeakers on the circular base plate are connected to the noise reduction circuit board by using the audio cables. On the third layer, there are a low voltage power supply and an infrared remote control switch that is convenient for remote control. The fourth layer is used as a cap for the whole device that makes it more complete. Thus, the whole structure of the V1.5 device is compact, firm, lightweight, portable, and suitable for remote control.

3.2. The Design of the Noise Reduction Device V2.0. In the case of the V2.0 device, the noise comes from the inner of the small closed space; thus, the device is designed to implement the noise reduction for the out open space. The structure and picture of the V2.0 device are shown in Figure 4. It has the similar structure as the V1.5 device and is composed of a circular chassis and three-layer clamping groove plates which are also joined together by four cylinders. For the V2.0 device, there are also eight clamping grooves made on the circular chassis. Four moving coil loudspeakers as the opposite phase output unit are set on four clamping grooves, while four 


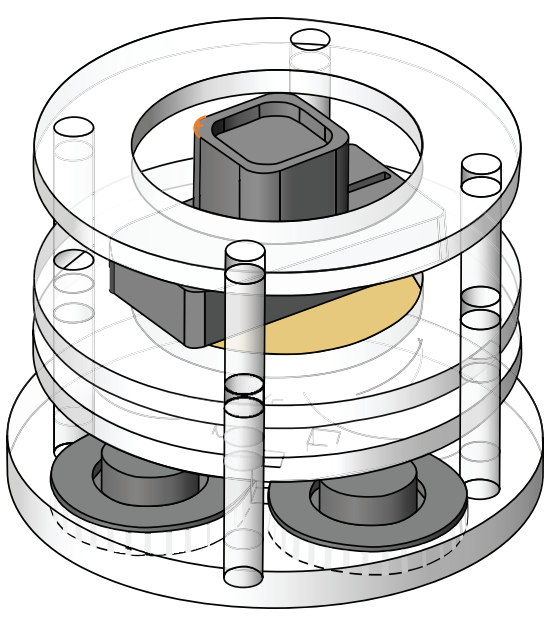

(a)

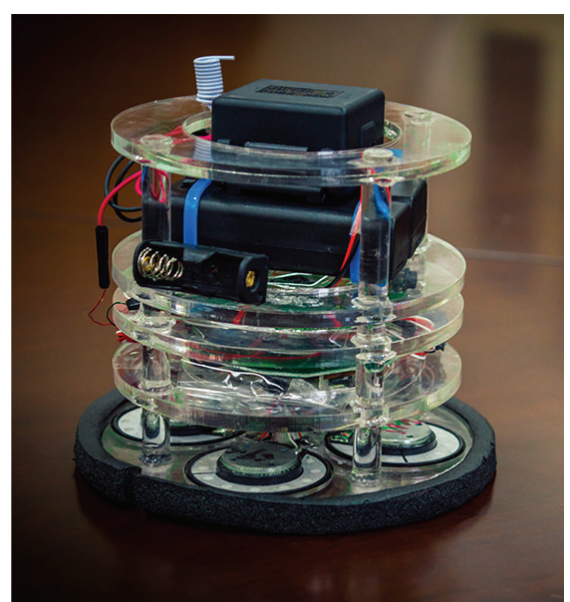

(b)

Figure 3: The structure (a) and picture (b) of the noise reduction device V1.5.

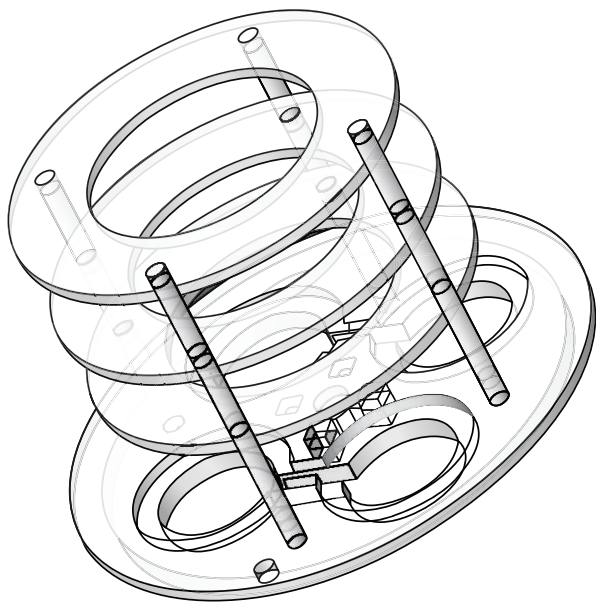

(a)

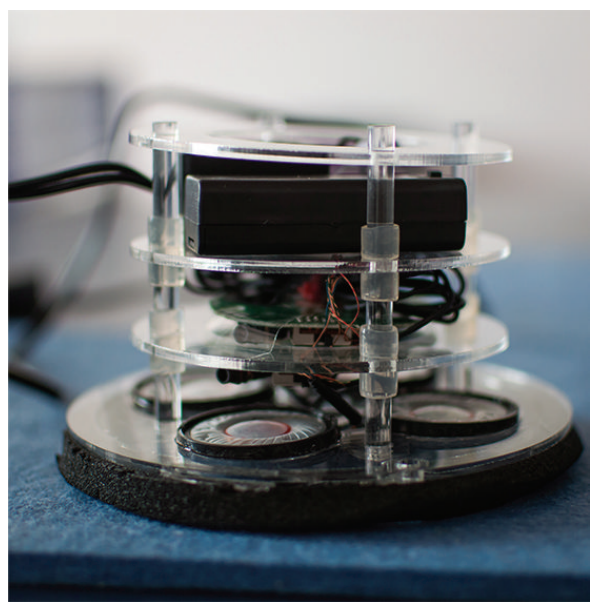

(b)

FIgURE 4: The structure (a) and picture (b) of the noise reduction device V2.0.

electret pickups as the noise collectors are mounted on the other grooves. Additionally, a noise reduction circuit board is fixed on the first layer of the clamping groove plates the same as the conditions of the V1.5 device. The pickup and loudspeaker on the circular chassis are also connected to the noise reduction circuit board through audio cable. On the second layer, there are a low voltage power supply and an infrared remote control switch. The third layer is also used as a cap for the noise reduction device. Compared with the V1.5 device, the V2.0 device is much smaller and lighter.

\section{Experimental Testing System and Method}

4.1. The Testing Platform for the V1.5 Device. Figure 5 shows the experimental testing system for the V1.5 device. In order to simulate small closed space, we designed a tubular space with an open end and a close end, which is surrounded by a hollow cylindrical organic glass casing and a sound insulating material wrapped on the outside of the casing. The tubular space has the length of $1 \mathrm{~m}$ and the inside diameter of $0.15 \mathrm{~m}$ that is equal to the outside diameter of the clamping groove on the bottom of the V1.5 device. When implementing the testing, the noise reduction device needs to be installed on the closed end of the tubular space, while a standard noise generator used as the noise source is set at 0.2 meter away from the noise reduction device. We then employ the decibel meter and the noise spectrum analyzer fixed in the tubular space through two frames to measure the changes in volume and frequency, respectively. Furthermore, the testing results from the decibel meter can be transferred by a data line to the analysis software in computer in real time.

We need to focus the analysis on two main variables: one is the frequency of the noise source and the other is the distance between the decibel meter probe and the noise reduction device. Thus, we address the following aspects: (1) the general effect on the noise reduction of this V1.5 device; (2) the impacts of the different frequencies on the noise reduction 


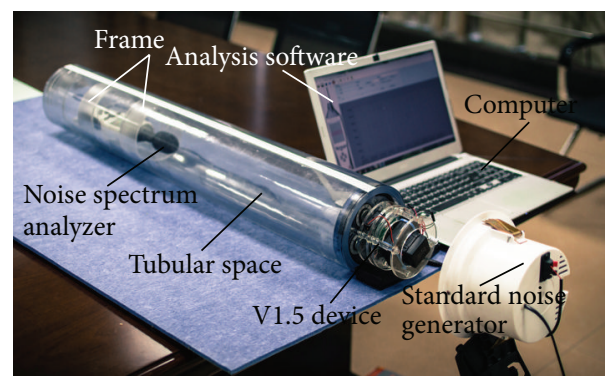

FIgURE 5: The picture of the experimental testing system for the noise reduction device V1.5.

effect; (3) the impacts of the various distances between the decibel meter probe and the noise reduction device on the noise reduction effect. We set up 12 testing points with an interval of $5 \mathrm{~cm}(5 \mathrm{~cm}, 10 \mathrm{~cm} \cdots 60 \mathrm{~cm})$ between the noise reduction device and the decibel meter probe. At each testing point, we measured the decibels at the 15 frequencies $(50 \mathrm{~Hz}$, $100 \mathrm{~Hz}, 150 \mathrm{~Hz} \cdots 500 \mathrm{~Hz}$, and $600 \mathrm{~Hz} \cdots 1000 \mathrm{~Hz}$ ) before and after the noise reduction processing, and the difference between the two groups of values can indicate the effect of the noise reduction device.

4.2. The Testing Platform for the V2.0 Device. The testing experimental system for the V2.0 device is shown in Figure 6. In order to simulate small open environment, we also designed a tubular space with an open end and a close end, which is surrounded by a hollow cylindrical organic glass casing and by a sound insulating material wrapped on the outside of the casing. The tubular space has the length of $0.6 \mathrm{~m}$ and the inside diameter of $0.15 \mathrm{~m}$. It is different from the V1.5 device that the V2.0 device needs to be installed on the open end of the tubular space, while a Bluetooth sound box employed as the noise source is fixed inside and on the bottom of the tubular space. The spectrum analyzer is supported by a tripod which can adjust the height in vertical direction and is connected to the computer through a data line, transferring the test results to the analysis software in real time. Accordingly, it is can be seen that the testing system for the V2.0 device has the fundamental difference from that for the V1.5 device. The spectrum analyzer is exposed in the open environment while the noise source is fixed in the closed tubular space. The HS5671B noise spectrum analyzer we used in the experiments adopts the capacitor microphone, the sensitivity of the spectrum analyzer is about $30 \mathrm{mV} / \mathrm{Pa}$, the frequency range is from $20 \mathrm{~Hz}$ to $\mathrm{KHz}$, and the measuring range is $25 \mathrm{~dB} \sim 130 \mathrm{~dB}(A), 25 \mathrm{~dB} \sim 130 \mathrm{~dB}(C)$, and $30 \mathrm{~dB} \sim$ $130 \mathrm{~dB}(Z)$, in which $A, C$, and $Z$ represent frequency weight.

Similar to testing system for V1.5 device, we select two main variables for analysis including the frequency of the noise source and the distance between the spectrum analyzer probe and the V2.0 device. In an open inverted cone space with the V2.0 device set at its vertex, we address the following aspects for analysis: (1) the general effect on the noise reduction of this V2.0 device; (2) the impacts of the different frequencies on the noise reduction effect; (3) the impacts of

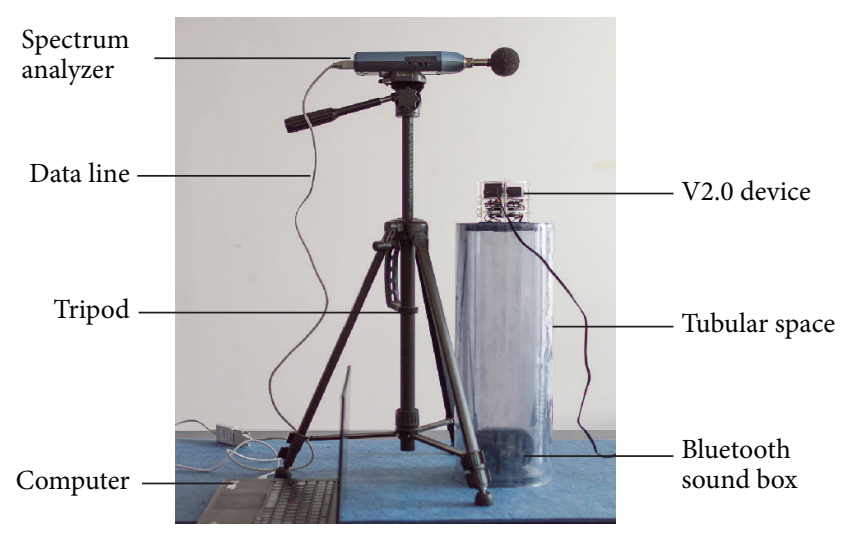

FIgURE 6: The picture of the experimental testing system for the noise reduction device V2.0.

the various distances between the decibel meter probe and the noise reduction device on the noise reduction effect; (4) the distribution of effect through the open inverted cone space. We establish a Cartesian coordinate system with the origin $(0,0)$ at the center of the pickups in the V2.0 device. The positive $y$ axis points along the vertical-upward, while the positive $x$ axis points along the horizontal-right. On the $y$ axis, we select 5 testing points with an interval of $5 \mathrm{~cm}((0,5)$, $(0,10) \cdots(0,25))$. In order to simulate the inverted cone space, the other three points including $(10,5),(20,10)$, and $(30,15)$ are added in this testing. According to the axis-symmetric principle, we use the test results of the three test points instead of the test results of conical surface. At each testing point, we measured the decibels with regard to 14 frequencies $(100 \mathrm{~Hz}, 150 \mathrm{~Hz} \cdots 500 \mathrm{~Hz}$, and $600 \mathrm{~Hz} \cdots 1000 \mathrm{~Hz})$ before and after noise reduction and use their differences to describe the noise reduction effect.

\section{The Performance Analysis on the Noise Reduction Devices}

\subsection{The Performance Analysis for the V1.5 Device}

5.1.1. The General Effect on the Noise Reduction at Low Frequency. Figure 7 gives the general effect on the noise reduction for the V1.5 device at low frequency. The decibel value of the noise reduction has a weak certain regularity with the increasing frequency. Two obvious peaks can be found in this curve at $250 \mathrm{~Hz}$ and $400 \mathrm{~Hz}$, where the effect values are $22 \mathrm{~dB}$ and $13 \mathrm{~dB}$, respectively. Both the values indicate far greater than that of the other frequencies. All the decibels with positive values suggest the feasibility and the correctness of our design.

5.1.2. The Impacts of the Distance on the Noise Reduction Effect. The impacts of the distance between the decibel meter probe and the noise reduction device on the noise reduction effect are shown in Figure 8. The $x$ axis gives 12 distances and the $y$ axis represents the decibel. Although the effects of 15 frequencies are obtained for each distance, for convenient analysis, we draw only 4 curves at the corresponding typical 


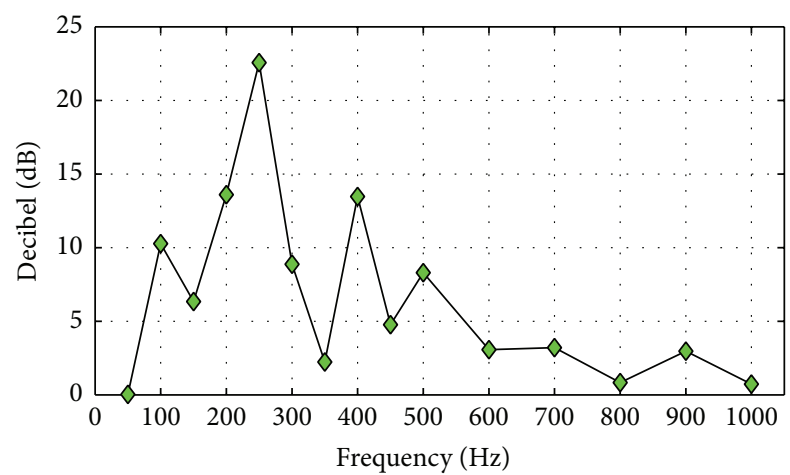

FIGURE 7: The general effect on the noise reduction for the V1.5 device at low frequency.

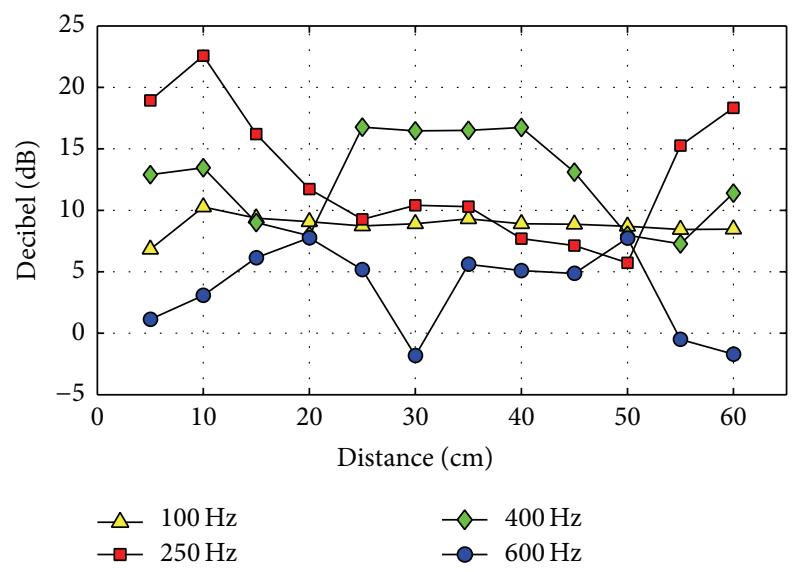

Figure 8: The impacts of the distance between the decibel meter probe and the noise reduction device on the noise reduction effect.

frequencies $(100 \mathrm{~Hz}, 250 \mathrm{~Hz}, 400 \mathrm{~Hz}$, and $600 \mathrm{~Hz})$ in Figure 8 . All the 15 curves, in general, have the slight decreasing trends with increasing distance after the first rise except that at $400 \mathrm{~Hz}$. This presents a weakening trend for the noise reduction effect with regard to the distance gradually. In the range of $0 \sim 25 \mathrm{~cm}$, the best effect for each frequency can be derived, while for the longer distances the effects show slight weakness. It is worth noting that the noise reduction effect of $250 \mathrm{~Hz}$ can reach $22.6 \mathrm{~dB}$ at about $10 \mathrm{~cm}$, which is far greater than the noise reduction effect of other frequencies at the same distance; for the range of $25 \sim 40 \mathrm{~cm}$, the effect curve at $400 \mathrm{~Hz}$ has an abnormal bulge with an extreme value of $16.7 \mathrm{~dB}$, which is also far greater than the effects at the other 14 frequencies at the same distance; when the distance increases more than $50 \mathrm{~cm}$, the effect at $250 \mathrm{~Hz}$ occurs a rising tail reaching $15 \mathrm{~dB}$. Accordingly, from the discussion above, a conclusion can be drawn that when the distance is less than $25 \mathrm{~cm}$, the most suitable frequency for noise reduction is $250 \mathrm{~Hz}$; for the distance falling in the range of $25 \sim 55 \mathrm{~cm}$, the most suitable frequency may be changed as $400 \mathrm{~Hz}$; regarding the distance more than $55 \mathrm{~cm}, 250 \mathrm{~Hz}$ can be the most suitable frequency for noise reduction again.

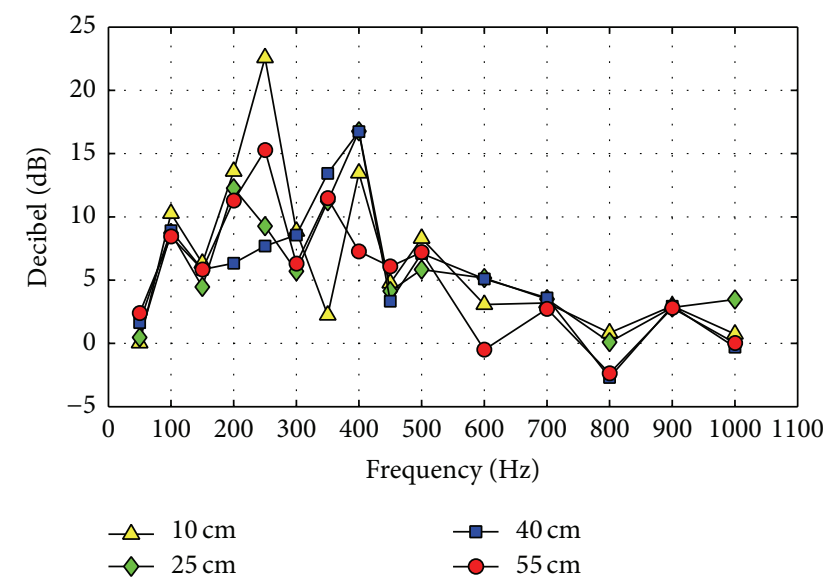

Figure 9: The curves of the noise reduction effect at different distances with regard to the frequency.

5.1.3. The Most Suitable Noise Reduction Distances for Different Frequencies. Figure 9 shows the curves of the noise reduction effect at different distances with regard to frequency. Similarly, the $x$ - and $y$-axis denote the frequency and the decibel value, respectively. Similar to Figure 8 , although the effects at 12 distances are tested in this experiment, for convenient analysis, we draw only 4 curves at the corresponding typical distances $(10 \mathrm{~cm}, 25 \mathrm{~cm}, 40 \mathrm{~cm}$, and $55 \mathrm{~cm})$ in Figure 9. The shapes of all the curves, in general, seem similar to the lognormal distribution, where the values experience the steep growth and then the slow decrease with the increasing frequency. Also, the noise reduction effect for all the curves at the range of $0 \sim 500 \mathrm{~Hz}$ is better than that in the other frequencies. In more detail, at the noise frequency ranging from 0 to $300 \mathrm{~Hz}$, the finest noise reduction effect can be derived at about $10 \mathrm{~cm}$ especially for $250 \mathrm{~Hz}$. For the noise with range of $300 \mathrm{~Hz}$ to $500 \mathrm{~Hz}$, the best effect can be obtained at about $40 \mathrm{~cm}$ especially for $400 \mathrm{~Hz}$. For the higher frequency more than $500 \mathrm{~Hz}$, the noise reduction effect decreases with increasing frequency at this four typical distances. It is noted that the noise reduction at $800 \mathrm{~Hz}$ is even negative; this may suggest that the noise reduction device makes primary noise slightly increased. We will research this anomaly in the further work.

\subsection{The Performance Analysis for the V2.0 Device}

5.2.1. The General Noise Reduction Effect along the Central Axis of the Inverted Cone Space. Figure 10 shows the general noise reduction effect of V2.0 device along the central axis of the inverted cone space. It is seen from Figure 10 that two peaks are found with $400 \mathrm{~Hz}$ as their boundary point. In the range of $100 \mathrm{~Hz}$ to $400 \mathrm{~Hz}$, the noise reduction effect with the first extreme value of $3.25 \mathrm{~dB}$ at $250 \mathrm{~Hz}$ has two shallow slopes on both sides. In the case of ranging from $400 \mathrm{~Hz}$ to $1000 \mathrm{~Hz}$, the curve with the second peak of $5.88 \mathrm{~dB}$ at $450 \mathrm{~Hz}$ has a steep growth and a slow down with increasing frequency. Generally, except for $100 \mathrm{~Hz}, 400 \mathrm{~Hz}$, and $1000 \mathrm{~Hz}$, the noise reduction effect of $\mathrm{V} 2.0$ device for the other 11 frequencies are 


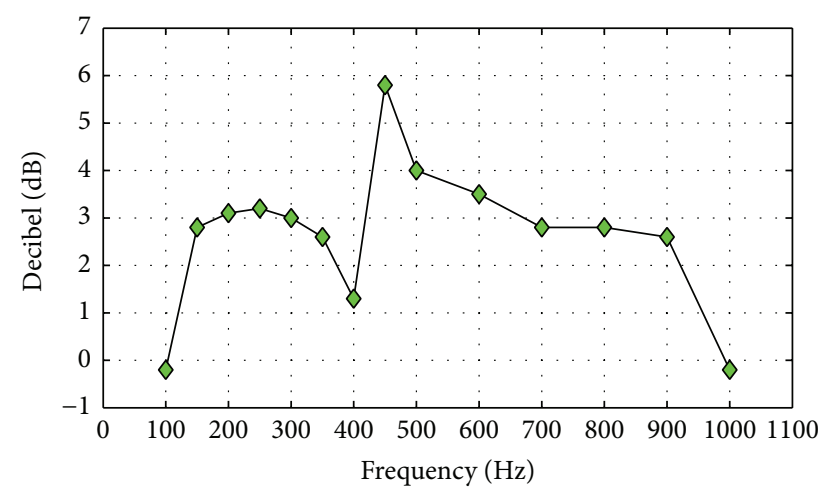

FIGURE 10: The general noise reduction effect for V2.0 device along the central axis of the inverted cone space.

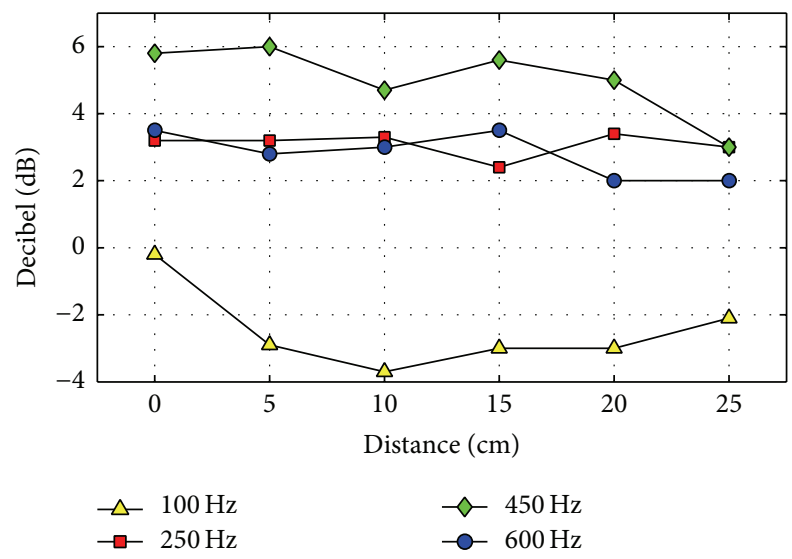

FIGURE 11: The noise reduction effect for V2.0 device varied with regard to the distance along the central axis of the inverted cone space.

larger than $2.5 \mathrm{~dB}$. It is unexpected that the noise reduction effect is 0 or even negative at $100 \mathrm{~Hz}$ and $1000 \mathrm{~Hz}$, respectively, that shows the obvious deviation from Figure 7 of V1.5 device for both the curve shape and peak frequency. This may be attributed to the different sensitivity of the loudspeaker to different frequencies, and we will carry out a detailed study on this issue in future work.

5.2.2. The Noise Reduction Effect Varied with regard to the Distance along the Central Axis. The noise reduction effect varied with regard to the distance along the central axis of the inverted cone space is shown in Figure 11.

The $x$ axis gives 6 distances and the $y$ axis denotes the decibel. Although the effects of 14 frequencies are tested for each distance, for convenient analysis, we draw only 4 curves at the corresponding typical frequencies $(100 \mathrm{~Hz}$, $250 \mathrm{~Hz}, 450 \mathrm{~Hz}$, and $600 \mathrm{~Hz}$ ) in Figure 11. All the 14 curves, in general, have the slight and smoothing decreasing trends with increasing distance. This presents a weakening trend for the noise reduction effect with regard to the distance gradually. It is evident from Figure 11 that the ideal noise reduction effect can be obtained for each frequency at the distance of $5 \mathrm{~cm}$ away from the V2.0 device. It is remarkable that the noise

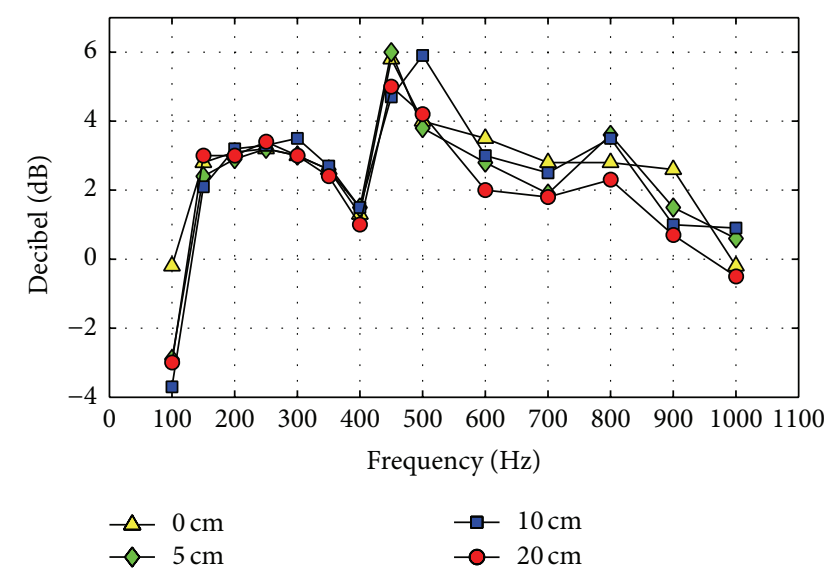

FIGURE 12: The noise reduction effect for V2.0 device varied with frequencies at different distances along the central axis of the inverted cone space.

reduction effect curve of $450 \mathrm{~Hz}$ is higher than that of other frequencies over all the distances, and the best effect of $6 \mathrm{~dB}$ at $450 \mathrm{~Hz}$ appears at $5 \mathrm{~cm}$. The noise reduction effect of $250 \mathrm{~Hz}$ is a fluctuation around $3.3 \mathrm{~dB}$ and the same as that for $600 \mathrm{~Hz}$. For $15 \mathrm{~cm}$ there is the lowest value $(2.6 \mathrm{~dB})$ for $250 \mathrm{~Hz}$ while the highest $(3.6 \mathrm{~dB})$ for $600 \mathrm{~Hz}$. In the range of $15 \mathrm{~cm}$ to $25 \mathrm{~cm}$, as the distance increases, the noise reduction effect of $250 \mathrm{~Hz}$ also increases while that of $600 \mathrm{~Hz}$ decreases. For the distance larger than $17 \mathrm{~cm}$, the noise reduction effect of $250 \mathrm{~Hz}$ is higher than that of $600 \mathrm{~Hz}$. Unexpectedly, the overall effect of $100 \mathrm{~Hz}$ is negative at any distance with the lowest value of $-3.8 \mathrm{~dB}$ at $10 \mathrm{~cm}$. It has a decrease before $10 \mathrm{~cm}$ and rises again subsequently. In conclusion, the most suitable frequency for noise reduction is $450 \mathrm{~Hz}$; and the effect is about $3 \mathrm{~dB}$ for both $250 \mathrm{~Hz}$ and $600 \mathrm{~Hz}$, while the noise reduction effect for $100 \mathrm{~Hz}$ is negative.

5.2.3. The Noise Reduction Effect Varied with Frequencies at Different Distances along the Central Axis. Figure 12 shows the noise reduction effect for V2.0 device varied with frequencies at different distances along the central axis of the inverted cone space. Similar to Figure 11, although the effects at 6 distances are tested in this experiment, for convenient analysis, we draw only 4 curves at the corresponding typical distances $(0 \mathrm{~cm}, 5 \mathrm{~cm}, 10 \mathrm{~cm}$, and $20 \mathrm{~cm})$ in Figure 12 . As seen from Figure 12, four curves are almost overlapping in the range of $150 \mathrm{~Hz}$ to $450 \mathrm{~Hz}$, illustrating that the differences of the noise reduction effect are small at the four distances. In the range of $450 \mathrm{~Hz}$ to $1000 \mathrm{~Hz}$, the noise reduction effect declines with distance in general. Compared with Figure 10, four curves all have two peaks at any distance with the $400 \mathrm{~Hz}$ as their common boundary point. Similarly, in the range of $100 \mathrm{~Hz}$ to $400 \mathrm{~Hz}$, the noise reduction effect with the first peak at $250 \mathrm{~Hz}$ has two shallow slopes on both sides. In the case of ranging from $400 \mathrm{~Hz}$ to $1000 \mathrm{~Hz}$, the curve with the second peak at $450 \mathrm{~Hz}$ has a steep growth and a slow down with increasing frequency. 


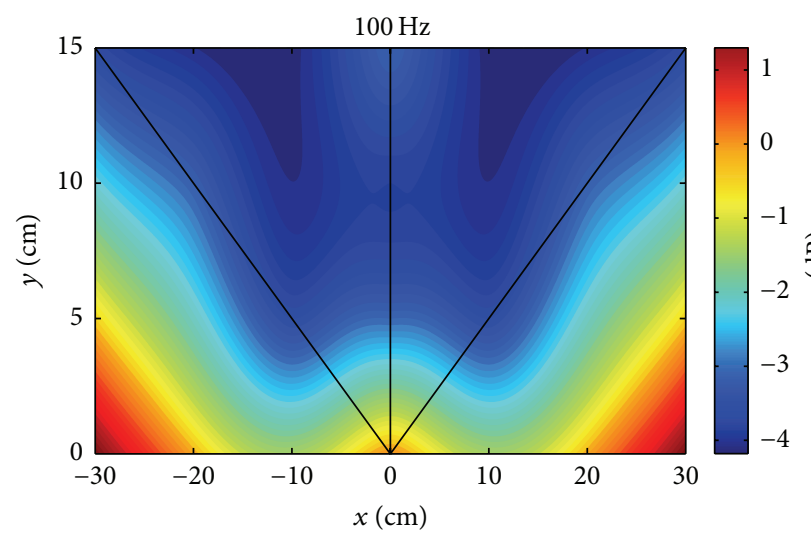

(a)

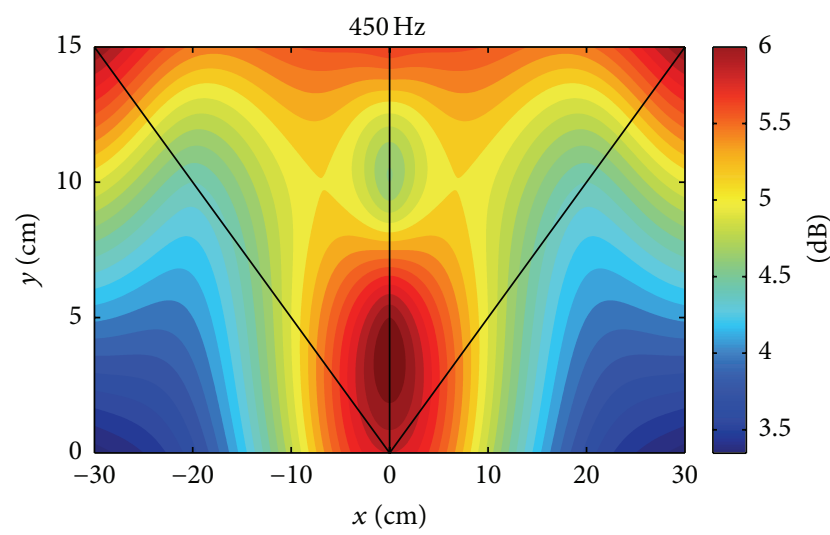

(c)

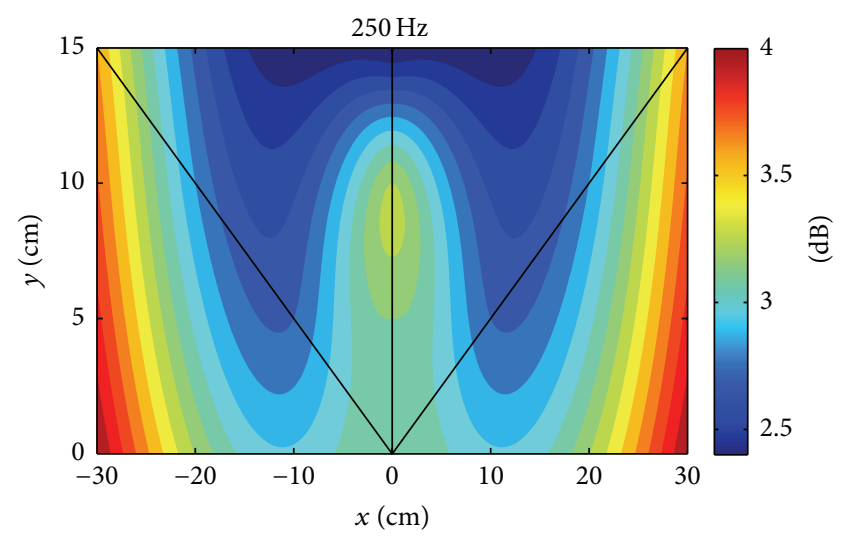

(b)

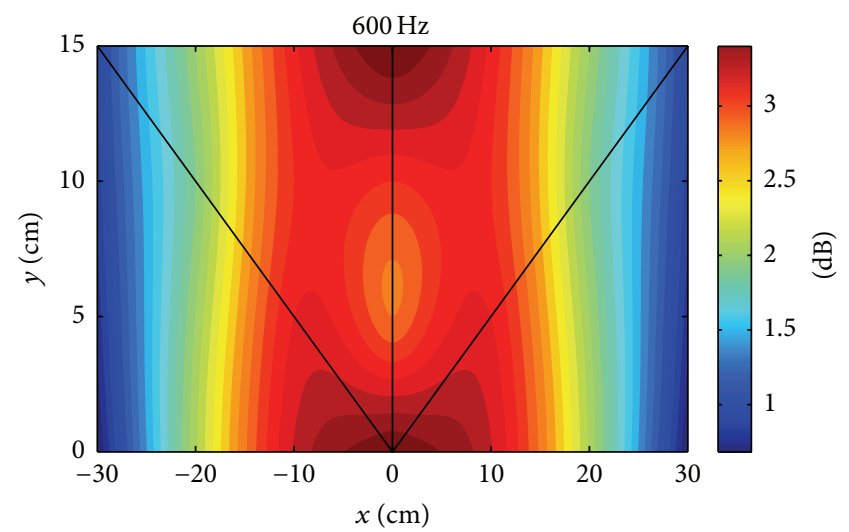

(d)

FIGURE 13: The distribution of noise reduction effect throughout the inverted cone space for $100 \mathrm{~Hz}, 250 \mathrm{~Hz}, 450 \mathrm{~Hz}$, and $600 \mathrm{~Hz}$, respectively.

5.2.4. The Distribution of Noise Reduction Effect throughout the Inverted Cone Space. Figures 13(a)-13(d) present the distribution of noise reduction effect throughout the inverted cone space for the typical $100 \mathrm{~Hz}, 250 \mathrm{~Hz}, 450 \mathrm{~Hz}$, and $600 \mathrm{~Hz}$, respectively. The origin $(0,0)$ denotes the position of the V2.0 device. The $x$ axis and $y$ axis represent distances of the testing points away from the origin in horizontal and vertical directions, respectively. The three black lines in each figure draw the central axis and envelope of the inverted cone space, and the color scale indicates the level of the noise reduction effect. It seems roughly that the noise reduction effect in each figure shows the obvious axisymmetric distribution with regard to the central axis or $y$ axis.

The noise reduction effect, shown in Figure 13(a), can weaken with increasing distance in the space. The highest value of $-1.1 \mathrm{~dB}$ at the origin indicates that the noise reduction effect for $100 \mathrm{~Hz}$ is not satisfied for our design. As seen from Figure 13(b), the noise reduction effect for $250 \mathrm{~Hz}$ is more than $2.5 \mathrm{~dB}$ inside the whole envelope of the cone space, and the best effect of $3.3 \mathrm{~dB}$ appears at $8 \mathrm{~cm}$ on the central axis. The effect, however, will decrease out away from the central axis. The decreasing rate above the center is faster than that of both sides; the slowest rate is beneath the center. In Figure 13(c), the noise reduction effect for $450 \mathrm{~Hz}$ is higher than $4.5 \mathrm{~dB}$ inside the whole envelope of the cone space with the maximum of $6.0 \mathrm{~dB}$ at $5 \mathrm{~cm}$ on the central axis. The noise reduction effect declines along the maximum center toward outside, while a minimum of $4.5 \mathrm{~dB}$ appears at $11 \mathrm{~cm}$ on the central axis. The noise reduction effect enhances along the minimum center toward outside; however, the effect weakens with space out the range of $10 \mathrm{~cm}$. In the range of $13 \sim 15 \mathrm{~cm}$, the noise reduction effect increases with regard to the increasing height in the vertical direction, and the high-value region appears on the upper edges. As shown in Figure 13(d), the noise reduction effect for $600 \mathrm{~Hz}$ is more than $1.3 \mathrm{~dB}$ in the whole cone space. The high-value region appears at the center of the upside-down cone space; the effect decreases along the central axis toward outside. There are two maximums of $3.4 \mathrm{~dB}$, respectively, appearing at the origin and $15 \mathrm{~cm}$ on the central axis, while the noise reduction effect decreases along this maximum toward outside.

5.3. Comparison between V1.5 and V2.0 Devices. As previously stated, the V1.5 device and the V2.0 device are both composed of circular chassis, clamping groove plate, cylinder, moving coil loudspeaker, electret pickup, noise reduction circuit board, low voltage power supply, and infrared remote control switch. The list of all the accessories used in the V1.5 and V2.0 devices is shown in Table 1. It is worth mentioning that there are four clamping groove plates and two noise 
TABLE 1: The list of all the accessories used in the V1.5 and V2.0 device.

\begin{tabular}{lcc}
\hline \multirow{2}{*}{ Accessory name } & \multicolumn{2}{c}{ Numbers } \\
& The V1.5 device & The V2.0 device \\
\hline Circular chassis & 1 & 1 \\
Clamping groove plate & 4 & 3 \\
Cylinder & 4 & 4 \\
Moving coil loudspeaker & 4 & 4 \\
Electret pickup & 4 & 4 \\
Noise reduction circuit board & 2 & 1 \\
Low voltage power supply & 1 & 1 \\
Infrared remote control switch & 1 & 1 \\
\hline
\end{tabular}

reduction circuit boards in the V1.5 device while there are only three clamping groove plates and one noise reduction circuit board in the V2.0 device.

Apart from the accessories used in the noise reduction device, there are also some different physical properties between the V1.5 device and the V2.0 device. For the V1.5 device, the height is $100 \mathrm{~mm}$ and the mass is $475 \mathrm{~g}$ while the V2.0 device is $15 \mathrm{~mm}$ shorter and $120 \mathrm{~g}$ lighter than the V1.5 device. The V1.5 device is applied in the small closed space, and the finest frequency for noise reduction is $250 \mathrm{~Hz}$ with the effect of $22 \mathrm{~dB}$. For the V2.0 device, it is applied in the small open space, and the finest frequency for noise reduction is $450 \mathrm{~Hz}$ with the effect of $5.88 \mathrm{~dB}$. The V2.0 device has the same physical properties as the V1.5 device in other aspects, which are shown in Table 2.

5.4. Discussion. In general, the V1.5 device can effectively eliminate the low-frequency noise in a small environment in some degree. According to the analysis, the noise reduction effect will decrease gradually with the increasing distance. It significantly relates with the spreading characteristics of the sound field in a small environment as well as the internal acoustic characteristics and sound insulation properties of the small space to some extent. In the course of practical use, we can assume that there is a specific-frequency noise and it could be eliminated effectively through changing the distance from the noise reduction device.

Compared with the V1.5 device, the V2.0 device has a great difference in the application environment and has poorer noise reduction effect. But there are still many similarities. The V2.0 device can eliminate the low-frequency noise effectively and stably in the open environment to a certain degree. In the inverted cone space, the optimal noise reduction effect appears on the central axis, and the noise reduction effect shows the obvious axisymmetric distribution in the inverted cone space. Meanwhile, on the central axis, the noise reduction effect presents a weakening trend with regard to the distance gradually while the noise reduction effect at a fixed distance changes with the noise frequency. These results can be attributed to the spreading characteristics of sound field in a small space. Then in the course of practical use, we can also assume that there is a specific-frequency noise and it could be eliminated through changing the location of the noise reduction device inside the space.

However, it is worth noting that the V1.5 and V2.0 device show negative effect values on some frequencies and distances. We need more repeated experiments to confirm these phenomena, which will be carried out as a detailed study in this issue in the further work.

\section{Conclusions}

Noise pollution has been given more and more attention due to the rapid development of modern industrial. The portable low-frequency noise reduction device we developed in this research can provide an effective way for solving low-frequency noise pollution problem in the small space. This work elaborates on the basic principle, the designing scheme, and the noise reduction effect of the noise reduction device. By means of ANC technology, the control elements and electroacoustic devices then produce a secondary sound wave. Since the secondary wave has the equal amplitude, opposite phase, and same propagating direction compared with the source ideally, the noise can be weakened to a lager extent along its propagating path based on the interference principle.

The noise reduction function is realized by using the integrated circuit which is made of a noise collector, a signal amplifier, an antialiasing filter, an A/D converter, an adaptive active noise controller, a D/A converter, a smoothing filter, an audio amplifier, and a reversed phase noise output module. For V1.5 device, we carried out three groups of experiment through building the tubular space with the length of $1 \mathrm{~m}$ and the inside diameter of $0.15 \mathrm{~m}$; and for V2.0 device, we also implemented four groups of experiment through the tubular space with the length of $0.6 \mathrm{~m}$ and the inside diameter of $0.15 \mathrm{~m}$. The results showed that (1) for V1.5 device, the noise reduction device can eliminate low-frequency $(50 \mathrm{~Hz} \sim$ $500 \mathrm{~Hz}$ ) noise effectively. The finest frequency for noise reduction is $250 \mathrm{~Hz}$ with the effect of $22 \mathrm{~dB}$. The noise reduction effect presents a weakening trend with regard to the distance. The low-frequency $(50 \mathrm{~Hz} \sim 300 \mathrm{~Hz})$ noise can be eliminated at the distance of $10 \mathrm{~cm}$ while the intermediatelow-frequency $(300 \mathrm{~Hz} \sim 500 \mathrm{~Hz}$ ) noise is $40 \mathrm{~cm}$. (2) For V2.0 device, the noise reduction device can undo low-frequency $(150 \mathrm{~Hz} \sim 600 \mathrm{~Hz})$ noise. The optimal frequency for noise reduction is $450 \mathrm{~Hz}$ with the effect of $5.88 \mathrm{~dB}$. Along the central axis of the inverted cone space, the noise reduction effect also presents a weakening trend with regard to the distance gradually. The low-frequency $(150 \mathrm{~Hz} 300 \mathrm{~Hz})$ noise can be removed at the distance of $10 \mathrm{~cm}$ while the intermediate-low-frequency $(300 \mathrm{~Hz} \sim 600 \mathrm{~Hz})$ noise is $20 \mathrm{~cm}$. The noise reduction effect shows the obvious axisymmetric distribution in the inverted cone space. We can avoid the disturbance of the high-frequency noise from outside in the experiments, since the high-frequency noise could produce inhibition effect. If some passive and insulation equipment is added in this device, the noise spectrum range can be extended from low frequency to high frequency. 
TABLE 2: Physical properties of the V1.5 and V2.0 device.

\begin{tabular}{|c|c|c|c|}
\hline \multirow{2}{*}{ Accessory name } & \multirow{2}{*}{ Physical property } & \multicolumn{2}{|c|}{ Parameter } \\
\hline & & The V1.5 device & The V2.0 device \\
\hline \multirow{7}{*}{ The noise reduction device } & Diameter of the circular chassis & $120 \mathrm{~mm}$ & $120 \mathrm{~mm}$ \\
\hline & Diameter of the clamping groove plate & $100 \mathrm{~mm}$ & $100 \mathrm{~mm}$ \\
\hline & Height & $100 \mathrm{~mm}$ & $85 \mathrm{~mm}$ \\
\hline & Mass & $475 \mathrm{~g}$ & $355 \mathrm{~g}$ \\
\hline & Applicable space & Small closed space & Small open space \\
\hline & The range of the noise reduction effect & $0 \sim 22 \mathrm{~dB}$ & $0 \sim 5.88 \mathrm{~dB}$ \\
\hline & The finest frequency for noise reduction & $250 \mathrm{~Hz}$ & $450 \mathrm{~Hz}$ \\
\hline \multirow{2}{*}{ Noise reduction circuit board } & Model & \multicolumn{2}{|c|}{ AS3420 } \\
\hline & Frequency range & \multicolumn{2}{|c|}{$20 \sim 20000 \mathrm{~Hz}$} \\
\hline \multirow{3}{*}{ Moving coil loudspeaker } & Model & \multicolumn{2}{|c|}{ JVCMX10 } \\
\hline & Diameter & \multicolumn{2}{|c|}{$40 \mathrm{~mm}$} \\
\hline & Frequency range & \multicolumn{2}{|c|}{$20 \sim 20000 \mathrm{~Hz}$} \\
\hline \multirow{4}{*}{ Electret pickup } & Model & \multicolumn{2}{|c|}{ PC-K100 } \\
\hline & Diameter & \multicolumn{2}{|c|}{$6 \mathrm{~mm}$} \\
\hline & Height & \multicolumn{2}{|c|}{$4 \mathrm{~mm}$} \\
\hline & Frequency range & \multicolumn{2}{|c|}{$20 \sim 20000 \mathrm{~Hz}$} \\
\hline Low voltage power supply & Voltage & \multicolumn{2}{|c|}{$1.5 \mathrm{~V}$} \\
\hline Infrared remote control switch & Model & \multicolumn{2}{|c|}{ JYS-ZHXXX12V1T1-100TM-2 } \\
\hline
\end{tabular}

\section{Conflict of Interests}

The authors declare that there is no conflict of interests regarding the publication of this paper.

\section{Acknowledgments}

The authors would like to thank the National Natural Science Foundation of China (no. 41304124), the basic research project in Jiangsu Province (BK20141480), and the Startup Foundation for Introducing Talent of NUIST for supporting this work.

\section{References}

[1] S. M. Kuo and D. R. Morgan, "Active noise control: a tutorial review," Proceedings of the IEEE, vol. 87, no. 6, pp. 943-973, 1999.

[2] S. J. Elliott, Signal Processing for Active Control, Academic Press, San Diego, Calif, USA, 2001.

[3] M. Latos and K. Stankiewicz, "Studies on the effectiveness of noise protection for an enclosed industrial area using global active noise reduction systems," Low Frequency Noise, Vibration and Active Control, vol. 34, no. 1, pp. 9-20, 2015.

[4] Z. H. Xu, C.-M. Lee, and Z. Qiu, "A study of the virtual microphone algorithm for ANC system working in audio interference environment," Journal of Low Frequency Noise, Vibration and Active Control, vol. 33, no. 2, pp. 189-206, 2014.

[5] P. Z. Zhang and Q. Z. Zhang, "A simplified active noise control of fuzzy neural network method," Journal of Changchun Institute of Technology, vol. 8, no. 1, pp. 21-23, 2007.

[6] P. Lueg, "Process of silence sound oscillation," US Patent, 1936.

[7] H. F. Olson and E. G. May, "Electronic sound absorber," The Journal of the Acoustical Society of America, vol. 25, no. 6, pp. 1130-1136, 1953.
[8] W. B. Conover, "Fighting noise with noise," Noise Control, vol. 2, no. 2, pp. 78-82, 1956.

[9] M. Salikuddin and B. T. Zinn, "Adaptation of the impedance tube technique for the measurement of combustion process admittances," Journal of Sound and Vibration, vol. 68, no. 1, pp. 119-132, 1980.

[10] K. L. Anderson, G. L. Blankenship, and L. G. Lebow, "Rulebased adaptive PID controller," in Proceedings of the 27th IEEE Conference on Decision and Control, pp. 564-569, December 1988.

[11] Q.-Z. Zhang, W.-S. Gan, and Y.-L. Zhou, "A model predictive algorithm for active control of nonlinear noise processes," Shock and Vibration, vol. 12, no. 3, pp. 227-237, 2005.

[12] S. Liu, L. Z. Wang, and B. Li, "An active noise reduction headphone with noise reduction controlling method and system," Chinese Patent CN201410854148, 2014.

[13] S.-P. Moon, J. W. Lee, and T.-G. Chang, "Performance analysis of an adaptive feedback active noise control based earmuffs system," Applied Acoustics, vol. 96, pp. 53-60, 2015.

[14] N. Miyazaki and Y. Kajikawa, "Head-mounted active noise control system with virtual sensing technique," Journal of Sound and Vibration, vol. 339, pp. 65-83, 2014.

[15] L. Zhang and X. Qiu, "Causality study on a feedforward active noise control headset with different noise coming directions in free field," Applied Acoustics, vol. 80, pp. 36-44, 2014.

[16] M. Guldenschuh and R. de Callafon, "Detection of secondarypath irregularities in active noise control headphones," IEEE/ACM Transactions on Audio, Speech, and Language Processing, vol. 22, no. 7, pp. 1148-1157, 2014.

[17] P. Wang, J. Tao, and X. Qiu, "Active noise control in the exhaust port of a vacuum cleaner," The Journal of the Acoustical Society of America, vol. 131, no. 4, pp. 3471-3479, 2012.

[18] J. Pan, Y. Liu, J. Lu, and X. Qiu, "Active control of noise in vehicle cabins," The Journal of the Acoustical Society of America, vol. 131, no. 4, pp. 3379-3385, 2012. 
[19] B. Kwon and Y. Park, "Interior noise control with an active window system," Applied Acoustics, vol. 74, no. 5, pp. 647-652, 2013.

[20] T. Airaksinen and J. Toivanen, "An optimal local active noise control method based on stochastic finite element models," Journal of Sound and Vibration, vol. 332, no. 26, pp. 6924-6933, 2013.

[21] N. Tanaka, "Local and global active noise control using a parametric array loudspeaker," The Journal of the Acoustical Society of America, vol. 134, no. 5, pp. 4189-4189, 2013.

[22] I. T. Ardekani and W. H. Abdulla, "Adaptive active control of free space acoustic noise," The Journal of the Acoustical Society of America, vol. 133, no. 5, pp. 3302-3314, 2013.

[23] I. T. Ardekani and W. H. Abdulla, "Active noise control in three dimensions," IEEE Transactions on Control Systems Technology, vol. 22, no. 6, pp. 2150-2159, 2014.

[24] M.-C. Chiu, "Acoustical treatment of multi-tone broadband noise with hybrid side-branched mufflers using a simulated annealing method," Journal of Low Frequency Noise Vibration and Active Control, vol. 33, no. 1, pp. 79-112, 2014.

[25] S. G. Hill, S. D. Snyder, and N. Tanaka, "Practical implementation of an acoustic-based modal filtering sensing technique for active noise control," Applied Acoustics, vol. 68, no. 11-12, pp. 1400-1426, 2007.

[26] C. D. Petersen, R. Fraanje, B. S. Cazzolato, A. C. Zander, and C. H. Hansen, "A Kalman filter approach to virtual sensing for active noise control," Mechanical Systems and Signal Processing, vol. 22, no. 2, pp. 490-508, 2008.

[27] M. Tarabini and A. Roure, "Modeling of influencing parameters in active noise control on an enclosure wall," Journal of Sound and Vibration, vol. 311, no. 3-5, pp. 1325-1339, 2008.

[28] Y. L. Zhou, Y. X. Yin, and Q. Z. Zhang, "Active control of repetitive impulsive noise in a non-minimum phase system using an optimal iterative learning control algorithm," Journal of Sound and Vibration, vol. 332, no. 18, pp. 4089-4102, 2013.

[29] M. Ferrer, M. de Diego, G. Piñero, and A. Gonzalez, "Active noise control over adaptive distributed networks," Signal Processing, vol. 107, pp. 82-95, 2015.

[30] V. Patel and N. V. George, "Nonlinear active noise control using spline adaptive filters," Applied Acoustics, vol. 93, pp. 38-43, 2015.

[31] L. Y. Yu, H. Y. Gao, Q. M. Xue, T. T. Yu, C. Wang, and W. H. Yan, "A portable low-frequency noise reduction device," Chinese patent, CN201420341696, 2014.

[32] W. H. Yan, H. Y. Gao, Q. M. Xue, L. Y. Yu, T. T. Yu, and C. Wang, "A low-frequency noise reduction device for small space," Chinese Patent CN201510068988X, 2015. 


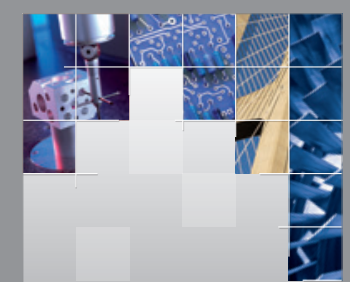

\section{Enfincering}
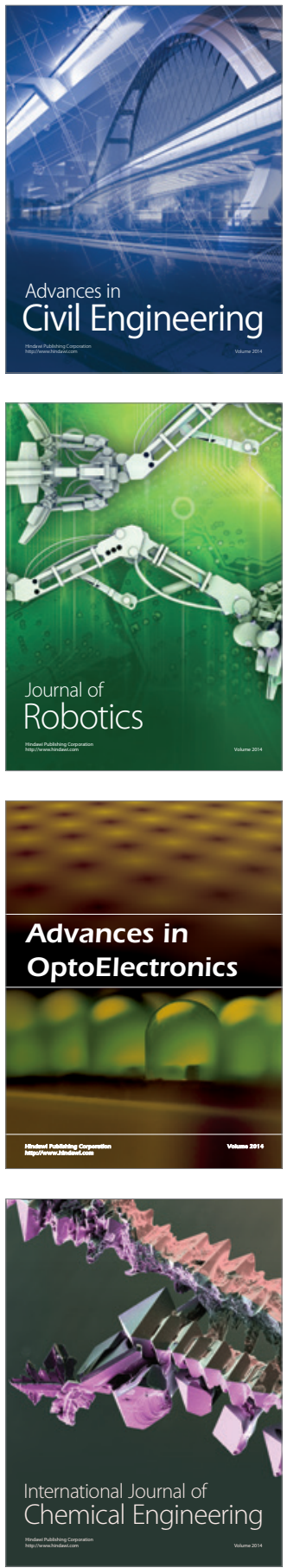

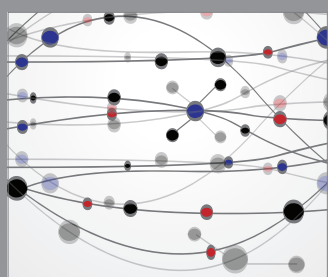

The Scientific World Journal

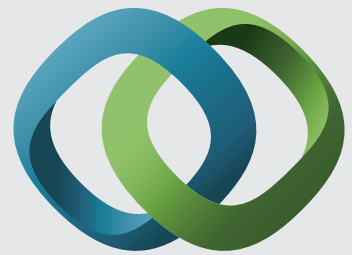

\section{Hindawi}

Submit your manuscripts at

http://www.hindawi.com
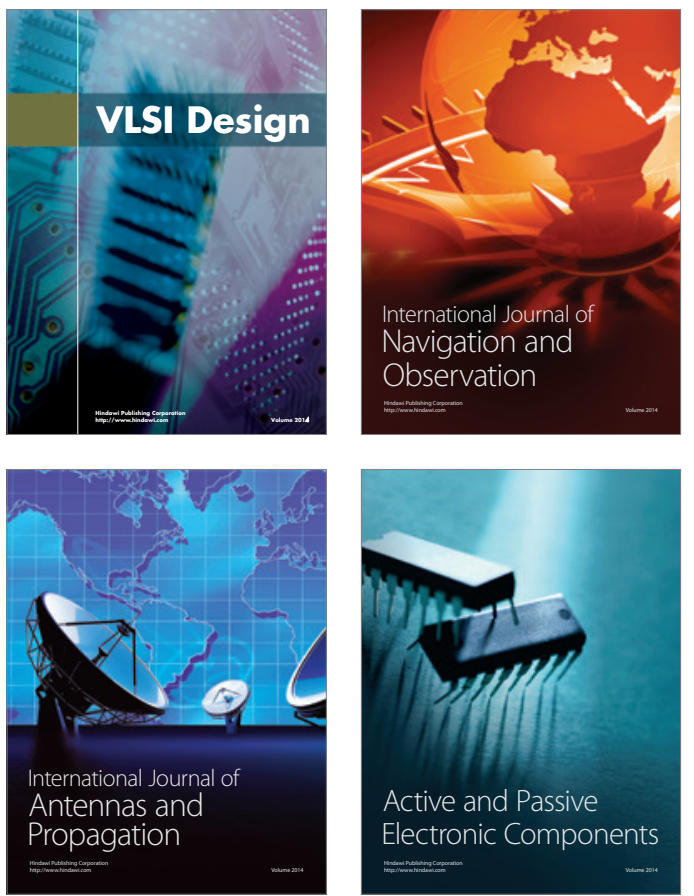
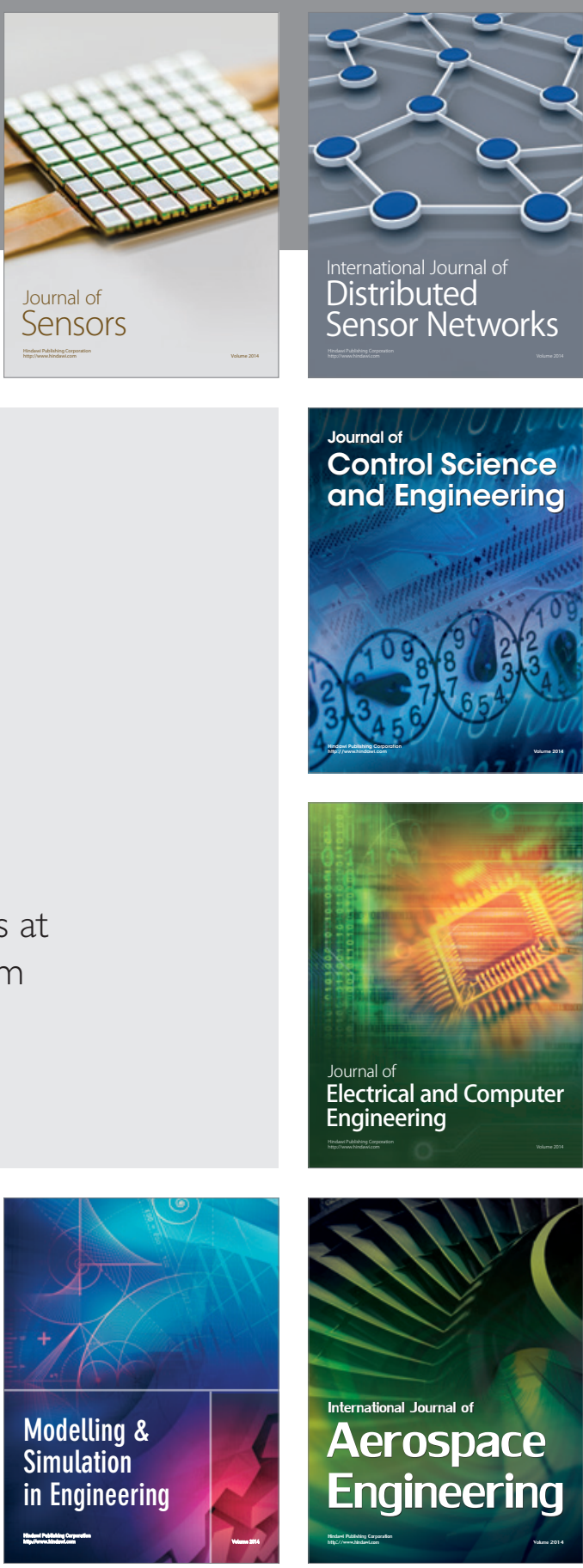

International Journal of

Distributed

Sensor Networks

Journal of

Control Science

and Engineering
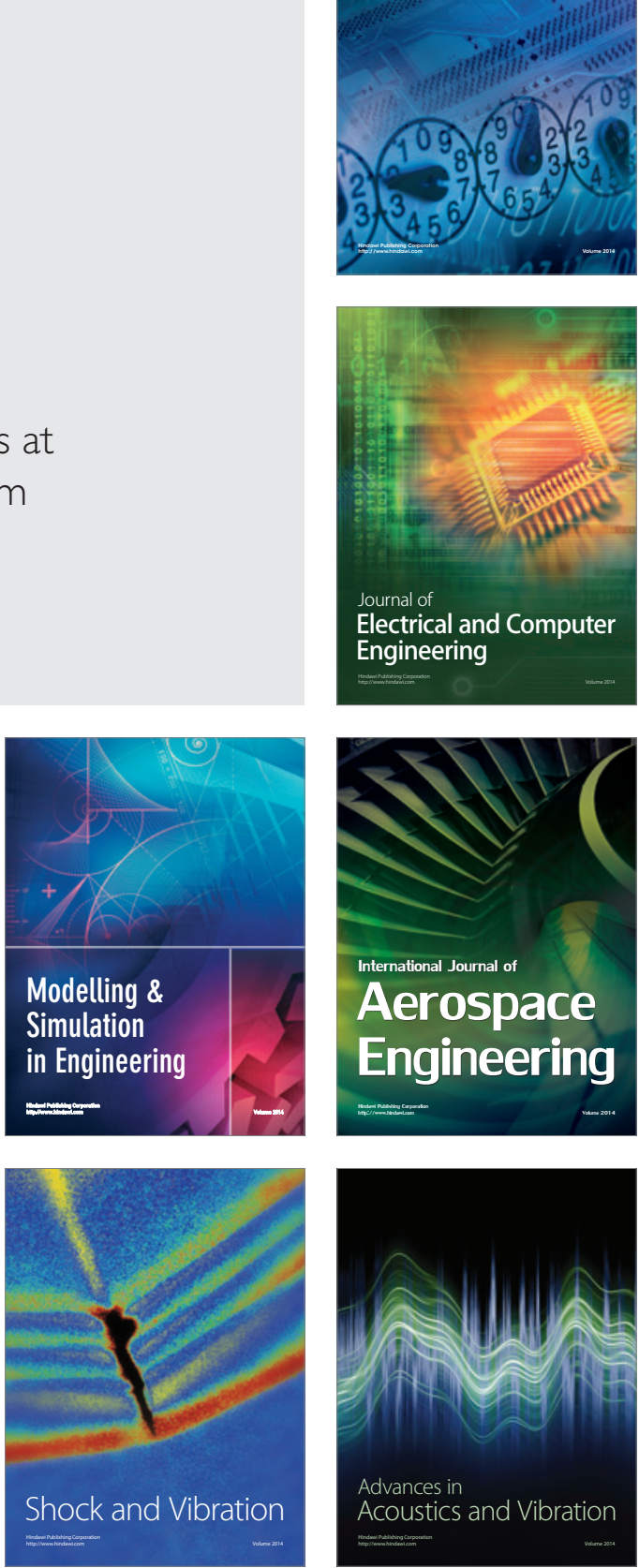